\title{
SHAPE EVOLUTION OF MASSIVE EARLY-TYPE GALAXIES: CONFIRMATION OF INCREASED DISK PREVALENCE AT $z>1$
}

\author{
Yu-Yen Chang ${ }^{1}$, Arjen van der WeL ${ }^{1}$, Hans-Walter Rix ${ }^{1}$, Stijn Wuyts $^{2}$, \\ Stefano Zibetti $^{3}$, Balasubramanian Ramkumar ${ }^{1}$, And BradFord Holden ${ }^{4}$ \\ ${ }^{1}$ Max Planck Institute for Astronomy, Koenigstuhl 17, D-69117 Heidelberg, Germany; chang@ mpia.de \\ ${ }^{2}$ Max Planck Institute for Extraterrestrial Physics, Postfach 1312, Giessenbachstr., D-85741 Garching, Germany \\ ${ }^{3}$ INAF-Osservatorio Astrofisico di Arcetri, Largo Enrico Fermi 5, I-50125 Firenze, Italy \\ ${ }^{4}$ UCO/Lick Observatory, Department of Astronomy and Astrophysics, University of California, Santa Cruz, CA 95064, USA \\ Received 2012 July 9; accepted 2012 November 8; published 2012 December 18
}

\begin{abstract}
We use high-resolution $K$-band VLT/HAWK-I imaging over $0.25 \mathrm{deg}^{2}$ to study the structural evolution of massive early-type galaxies since $z \sim 2$. Mass-selected samples, complete down to $\log \left(M / M_{\odot}\right) \sim 10.7$ such that "typical" $\left(L^{*}\right)$ galaxies are included at all redshifts, are drawn from pre-existing photometric redshift surveys. We then separate the samples into different redshift slices and classify them as late- or early-type galaxies on the basis of their specific star formation rate. Axis-ratio measurements for the $\sim 400$ early-type galaxies in the redshift range $0.6<z<1.8$ are accurate to 0.1 or better. The projected axis-ratio distributions are then compared with lower redshift samples. We find strong evidence for evolution of the population properties: early-type galaxies at $z>1$ are, on average, flatter than at $z<1$ and the median projected axis ratio at a fixed mass decreases with redshift. However, we also find that at all epochs $z \lesssim 2$, the most massive early-type galaxies $\left(\log \left(M / M_{\odot}\right)>11.3\right)$ are the roundest, with a pronounced lack of galaxies that are flat in projection. Merging is a plausible mechanism that can explain both results: at all epochs, merging is required for early-type galaxies to grow beyond $\log \left(M / M_{\odot}\right) \sim 11.3$, and all early types over time gradually and partially lose their disk-like characteristics.
\end{abstract}

Key words: cosmology: observations - galaxies: elliptical and lenticular, cD - galaxies: evolution - galaxies: formation - galaxies: structure

Online-only material: color figures, machine-readable table

\section{INTRODUCTION}

In our theory of galaxy formation, the initial angular momentum, radiative energy loss of the gas, and some degree of angular momentum conservation result in gas settling into disks before most of the stars form, which makes for oblate, rotating stellar systems. Observationally, all but the most massive galaxies in the present-day universe have a disk-like structure and are rotating (Dressler 1980; Kormendy \& Djorgovski 1989; Marinoni et al. 1999; Krajnović et al. 2008; Holden et al. 2009; Emsellem et al. 2011; Cheng et al. 2011).

Nonetheless, even in disk-like, rotating galaxies, many stars reside in pressure-supported bulges. Photometric decompositions indicate that in the present-day universe $58 \% \pm 7 \%$ of stars are in the spheroids and $42 \% \pm 7 \%$ are in the disks (Benson et al. 2007). Violent mergers are thought to scramble the orbits that originally were formed and lived in disks (Toomre 1977; White \& Rees 1978; Joseph \& Wright 1985; Schweizer et al. 1990; Kauffmann et al. 1993; Steinmetz \& Navarro 2002). This process dominates the evolution of the most massive galaxies, which do not show evidence for disks and are generally round and entirely supported by pressure instead of rotation (Jorgensen \& Franx 1994; Vincent \& Ryden 2005; van der Wel et al. 2009b, hereafter vdW09; Bernardi et al. 2011; Emsellem et al. 2011; Holden et al. 2012, hereafter H12).

In this paper, we use a low specific star formation as the definition of an early-type galaxy, motivated by the smooth appearance of the light profiles of galaxies with little or no young stars or (star-forming) gas. Early-type galaxies show a relatively abrupt change in their structure as a function of galaxy mass.
vdW09 and H12 show that galaxies with $\log \left(M / M_{\odot}\right)<11$ have a broad projected axis-ratio distribution, indicative of a disk-like stellar body (with a typical short-to-long intrinsic axis ratio of about 1:3). In contrast, early-type galaxies with $\log \left(M / M_{\odot}\right)>11$ rarely appear highly flattened, suggesting that their formation channel destroyed any pre-existing stellar disks, with "dry" merging between generally gas-poor progenitors as a very plausible mechanism.

Such "dry" mergers can reconcile the relatively late, and continuous, assembly of massive early types with their old, passively evolving stellar populations. In addition, the observed small radii and high densities of early-type galaxies at redshifts $z>1$ (e.g., van Dokkum et al. 2008; van der Wel et al. 2008) can also be explained by "dry" merging (e.g., Khochfar \& Silk 2006; van der Wel et al. 2009a; Hopkins et al. 2010). Minor mergers are plausibly the driver of size growth, given that major mergers are less efficient in "puffing up" galaxies (e.g., Bezanson et al. 2009), such that major merging would overproduce the number of massive galaxies in the present-day universe (e.g., McLure et al. 2012). Given the strong evidence for continuous evolution of the number of early-type galaxies (e.g., Bell et al. 2004; Faber et al. 2007) and size evolution, one may expect that their structural properties also evolve, especially if merging is invoked as an evolutionary mechanism. To test this, H12 compared the axis-ratio distribution of a large sample of early-type galaxies at $z \sim 0.7$ with that of the local population, but found no evidence for shape evolution for masses larger than $3 \times 10^{10} M_{\odot}$. However, there could be shape evolution at higher redshifts, as major merging occurred more frequently at early epochs (e.g., Robaina et al. 2010) and the early-type galaxy number was much lower at $z>1$ than it is today. 
Van der Wel et al. (2011, hereafter vdW11) showed that a good portion of a small sample of $z \sim 2$ early-type galaxies appeared flat in projection, indicative of a disk-like structure. Chevance et al. (2012) and Buitrago et al. (2012) point out that existing samples are too small to confirm or rule out evolution in the axis-ratio distribution, but they attest, based on the Sérsic index distribution, that these galaxies have more disklike structural properties than present-day early-type galaxies. Recently, Bruce et al. (2012) used bulge-disk decompositions of massive early-type galaxies at $z>1$ to show that many of them host pronounced disks. Whitaker et al. (2012) show a hint of an evolving axis-ratio distribution of early-type galaxies out to $z \sim 1.5$, but these authors did not explore this in detail.

In this paper, we explore the (projected) shapes for a large sample ( $\sim 400$ objects) of early-type galaxies, selected to have masses $\log \left(M / M_{\odot}\right)>10.7$ and low star formation rates $\left(\mathrm{sSFR}<1 / 3 t_{\mathrm{H}}(z)\right.$, see Section 2.5.), which were drawn from a wide, high-resolution, near-infrared ( $K$-band) imaging mosaic from the Very Large Telescope (VLT)/HAWK-I; we investigate whether early-type galaxies at $z>1$ show evolution in structure compared to present-day counterparts. Specifically, we will address the question of whether $z>0.8$ early-type galaxies are more or less disk-like than at the present epoch, and whether early-type galaxies at those epochs also become rounder with increasing mass, as seen today.

The structure of this paper is as follows. In Section 2, we describe the data and select our sample of early-type galaxies. In Section 3, we analyze the projected axis-ratio distribution and its evolution since $z \sim 2$. In Section 4 , we summarize our results.

In this paper, we use $\mathrm{AB}$ magnitudes and adopt the cosmological parameters $\left(\Omega_{M}, \Omega_{\Lambda}, h\right)=(0.3,0.7,0.7)$.

\section{DATA}

The first step is to compile a catalog with photometric redshifts and stellar masses of galaxies in the extended Chandra Deep Field South (ECDFS) from the Multiwavelength Survey by Yale-Chile (MUSYC; Taylor et al. 2009; Cardamone et al. 2010). Then we use high-resolution VLT/HAWK-I $K$-band imaging available over essentially the full ECDFS (ESO program ID: 082.A-0890) to determine structural parameter (sizes, Sérsic indices, and projected axis ratios) for these galaxies. The high fidelity of these measurements, verified through a comparison with results from Hubble Space Telescope (HST) imaging, allows us to select early-type galaxies up to $z=1.8$.

\subsection{Multiwavelength Data and SED Fitting}

MUSYC compiled observations in 32 bands, ranging from the UV to the near-infrared for the ECDFS, for which Cardamone et al. (2010) provide an optically selected catalog which we use here. We use the method and algorithms described by Wuyts et al. (2011b) to infer photometric redshifts, stellar masses, and rest-frame colors. Briefly, to estimate photometric redshifts ( $z_{\text {photo }}$ ) we use EAzY (Brammer et al. 2008), and to estimate stellar masses, star formation rates, and rest-frame colors we use FAST (Kriek et al. 2009). We only include objects with significant detection of $J-, H$-, and $K$-band imaging, and reject stars by choosing only objects with $J-K>0.05$. We adopt the Bruzual \& Charlot (2003) model and a Chabrier (2003) stellar initial mass function. A range of ages, star formation histories, and extinction parameters are explored. This parent catalog contains 19,642 objects.

H12 independently determined the stellar masses of earlytype galaxies in the ECDFS in the redshift range $0.6<z<0.8$. Those mass estimates are designed to match the stellar mass estimates of present-day early-type galaxies. Since we aim to do the same, we add 0.1 dex to all our stellar mass estimates to correct for the median difference between the galaxies that are included in both our parent sample and the H12 sample. This correction is likely incorrect for star-forming galaxies, but those are not considered in this work. A full investigation of the absolute mass scale for $z>1$ is beyond the scope of this paper.

Star formation rates (SFRs) were derived following the procedures outlined in Wuyts et al. (2011a). Briefly, the unobscured SFR traced by the $U V$ was added to the dust-reemitted SFR inferred from FIDEL $24 \mu \mathrm{m}$ photometry (Magnelli et al. 2009) for $24 \mu \mathrm{m}$-detected sources, and for sources without $24 \mu \mathrm{m}$ detection a dust-corrected SFR was derived from stellar population modeling of the U-to- $8 \mu \mathrm{m}$ spectral energy distribution (SED).

\subsection{High-resolution, Near-infrared VLT/HAWK-I Imaging}

High-resolution $K$-band imaging from VLT/HAWK-I is central to our study to provide the structural parameters. We have obtained $1 \mathrm{hr}$ exposures for each of 16 adjacent tiles in a $30^{\prime} \times 30^{\prime}$ mosaic that covers the full ECDFS (Taylor et al. 2009), which is coincident with the HST/ACS coverage from GEMS (Galaxy Evolution from Morphologies and SEDs; Rix et al. 2004). Observation and reduction of the HAWK-I images were performed by S.Z. using a customized pipeline based on the original version distributed by ESO. Most notably, we implemented improved recipes for the construction of the master flat field and for the frame coaddition, which properly take into account object masks and variance maps. The new effective mask implementation in particular eliminates the effects of background over-subtraction which are often seen in connection with (bright) sources. The $5 \sigma$ point source limit is $K(\mathrm{AB})=24.3$, and the point spread function (PSF) has an FWHM of 0.'5 or smaller across the field. At that resolution, this ground-based $K$-band imaging allows us to quantify the rest-frame optical structural properties of $z>1$ galaxies with spatial resolution that differs by no more than a factor of $\sim 2$ from that obtained with $H S T /$ WFC3 in the $H$ band (see Figure 1). The image quality of our data is substantially better than the $\sim 0^{\prime \prime} 6-00^{\prime} .7$ seeing data used by Whitaker et al. (2012).

\subsection{Galaxy Structural Parameters}

We use GALAPAgOS (Galaxy Analysis over Large Areas: Parameter Assessment by GALFITting Objects from SExtractor; Barden et al. 2012) to separately process each of the 16 HAWK-I tiles. Here we briefly describe the process as relevant for the present study. For a full description, see Barden et al. (2012). GALAPAGOS first constructs a catalog with SExtractor (software for source extraction; Bertin \& Arnouts 1996). We choose the SExtractor detection parameters such that the catalog is only complete down to $K(\mathrm{AB})=23$; as we will demonstrate below, this is well beyond the limit down to which structural parameters can accurately be determined. GALAPAGOS then creates image and noise cutouts for each object, including neighboring objects as necessary. The noise map is obtained from the variance map that was produced for each of the HAWK-I tiles. The background is estimated for each object by identifying a set of sky pixels that are not influenced by any of the objects in the catalog. 


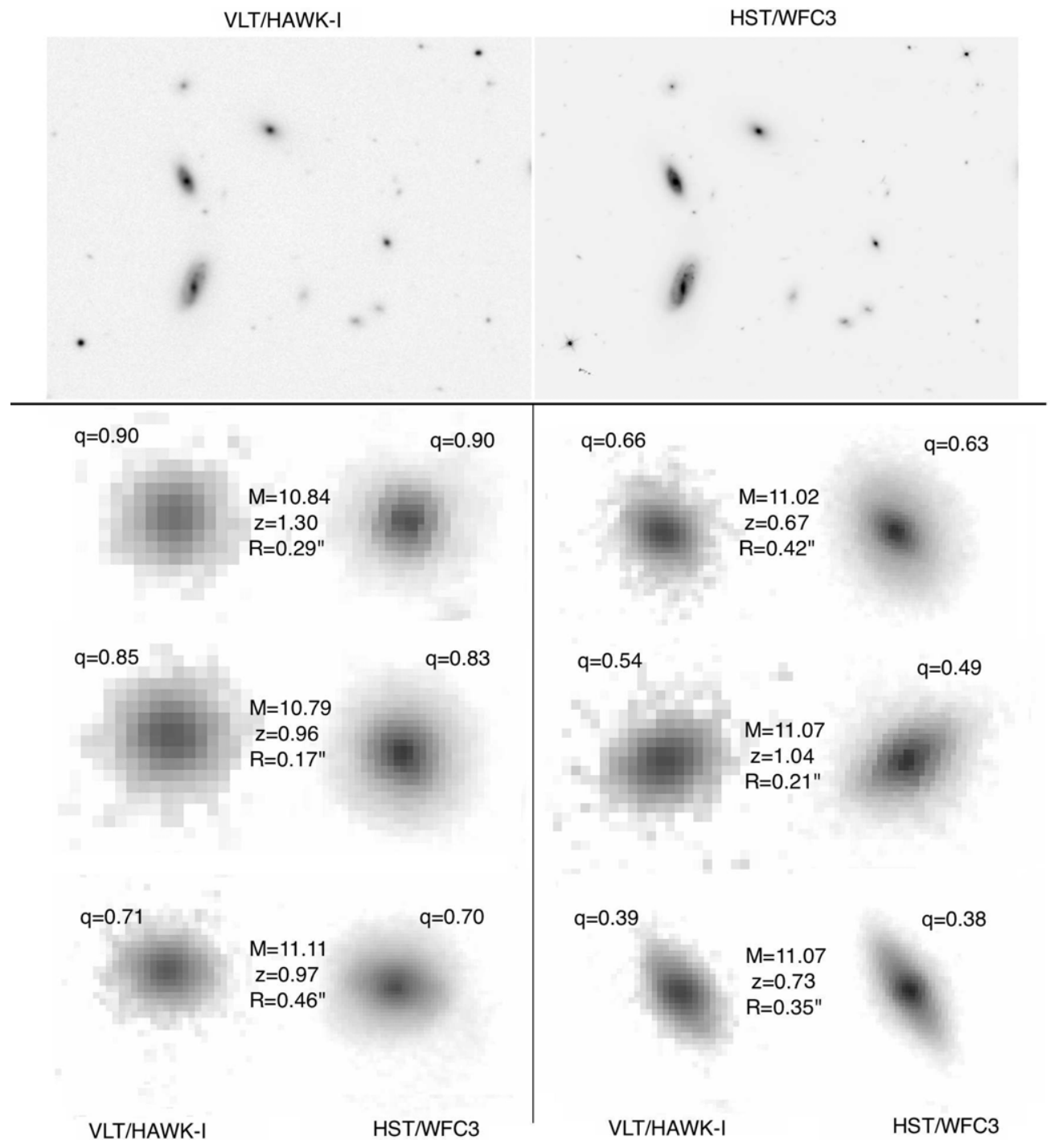

Figure 1. Comparison between VLT/HAWK-I imaging (top left) used for the analysis in this paper and HST/WFC3 imaging (top right; ERS data from Windhorst et al. 2011) of the same galaxies. The bottom panels show zoomed-in versions of the images of the HAWK-I and WFC 3 images of six early-type galaxies in the redshift, stellar mass range of interest, and circularized half-light radii of HAWK-I, as indicated. Each panel is about $2^{\prime \prime}$ on a side. These examples indicate that the resolution and depth of the HAWK-I imaging are sufficient to infer the projected axis ratio $q$, consistent with the values obtained from the WFC 3 images.

For each of the 16 tiles, a single star is taken as the PSF, chosen among the 5 brightest, isolated stars in each tile. This choice is made after subtracting a flux-scaled version of each of those 5 stars from $\sim 25$ stars in a tile and examining the residuals from this fit. The star that produces the cleanest residuals is selected as the PSF for that tile.

Then GALFIT (v3.0.3; Peng et al. 2002, 2010a) is called to perform the actual measurement of the structural parameters. A single Sérsic profile is fit to each target object. Neighboring objects are either masked or fit simultaneously. The free parameters in the fit are position, magnitude $(m)$, effective radius as measured along the major axis $\left(R_{e}\right)$, Sérsic index $(n)$, axis ratio $(q)$, and position angle. The input values of these parameters are taken from the SExtractor catalog (with the exception of $n$, for which 2.5 is adopted).

\subsection{Precision and Accuracy of Axis-ratio Measurements}

Our ability to determine axis ratios for distant galaxies is the limiting factor in our study. To establish the precision and accuracy of our measurements, we compare the axis ratios inferred from the HAWK-I imaging with those from GEMS (Häussler et al. 2007) for galaxies in the redshift range $0.6<z<$ 0.8 (the sample from H12). The wavelength difference between GEMS ( $z$ band) and HAWK-I ( $K$ band) could cause intrinsic differences between axis-ratio measurements that cannot be attributed to measurement errors. For this reason, we also compare these data with the axis-ratio estimates from objects in our sample that are contained with $H S T /$ WFC3 F160W imaging from Early Release Science (ERS) (Windhorst et al. 2011). GALAPAGOS is deployed in a similar fashion as described above-full details provided by van der Wel et al. (2012). Through the comparison with HST/ACS and HST/WFC3 (see Figure 2), we find that the precision of our HAWK-I axis-ratio estimates is better than $10 \%$ for galaxies brighter than $K(\mathrm{AB})=$ 22. In addition, we see no systematic difference between $H S T$ and VLT measurements shown in Figure 2. The differences in the median are -0.019 and -0.025 , respectively. Our axisratio measurements remain accurate over the entire magnitude range of our sample. In Figure 3 we show that, in addition, the accuracy of our axis-ratio measurements does not depend on redshift. 

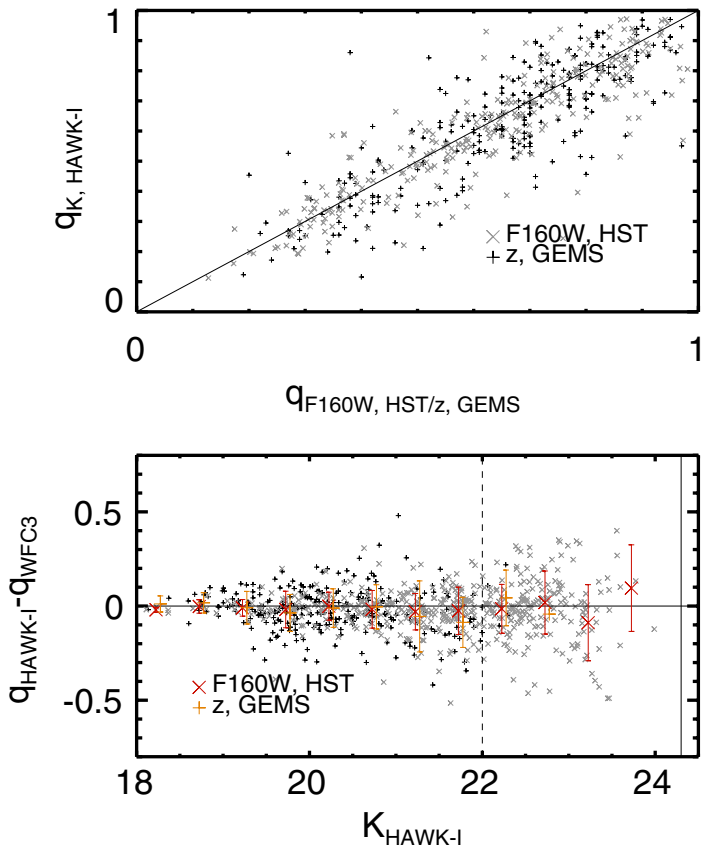

Figure 2. Comparison of axis-ratio measurements from the HAWK-I $K$ band and $H S T$ imaging. The crosses represent the comparison with measurements from WFC3 F160W imaging for a HAWK-I $K$-band-selected sample (regardless of galaxy type and redshift). The plus signs represent the comparison with measurements from HST/ACS F850LP of the H12 sample, which includes early-type galaxies in the redshift range $0.6<z<0.8$. The top panel directly compares the VLT and HST axis-ratio measurements; the bottom panel shows the difference between the VLT and HST measurements as a function of HAWK-I $K$-band magnitude. The red and orange bars represent the median and standard deviation for a series of magnitude bins. The standard deviation represents the measurement uncertainty in the VLT-inferred axis ratio, assuming the HST-based value as "truth." For galaxies, regardless of type, brighter than $K=22$ the uncertainty is smaller than or equal to 0.1 , and we adopt this as the magnitude limit for our study. Interestingly, the accuracy of the axisratio measurement is good down to at least $K \sim 24$; systematic effects in the axis-ratio measurements are small compared to the random uncertainty.

(A color version of this figure is available in the online journal.)

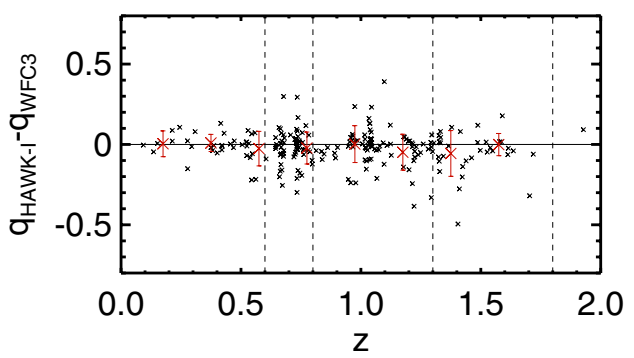

Figure 3. Comparison of axis-ratio measurements from VLT/HAWK-I and $H S T /$ WFC3 imaging as a function of redshift. The difference in axis-ratio measurements, $q_{\mathrm{HAWK}-\mathrm{I}}-q_{\mathrm{WFC} 3}$, has been multiplied by the total $K$-band flux in units of the $K$-band flux for a $K=21 \mathrm{object}$, such that variations in magnitude and their effect on the measurement uncertainty are accounted for. For galaxies in the narrow magnitude range (here, $20<K<22$ ), there is no systematic redshift dependence; the uncertainty for galaxies at $0.5<z<1.0$ is comparable to the uncertainty for galaxies at $z>1$. The trends shown in Figure 2 and this figure imply that the accuracy of our axis-ratio measurements is $10 \%$ or better for all galaxies in our sample.

(A color version of this figure is available in the online journal.)

\subsection{Sample Selection}

We match the MUSYC-based catalog (containing photometric redshifts and stellar population properties) with the HAWKI-based catalog (containing $K$-band magnitudes and structural

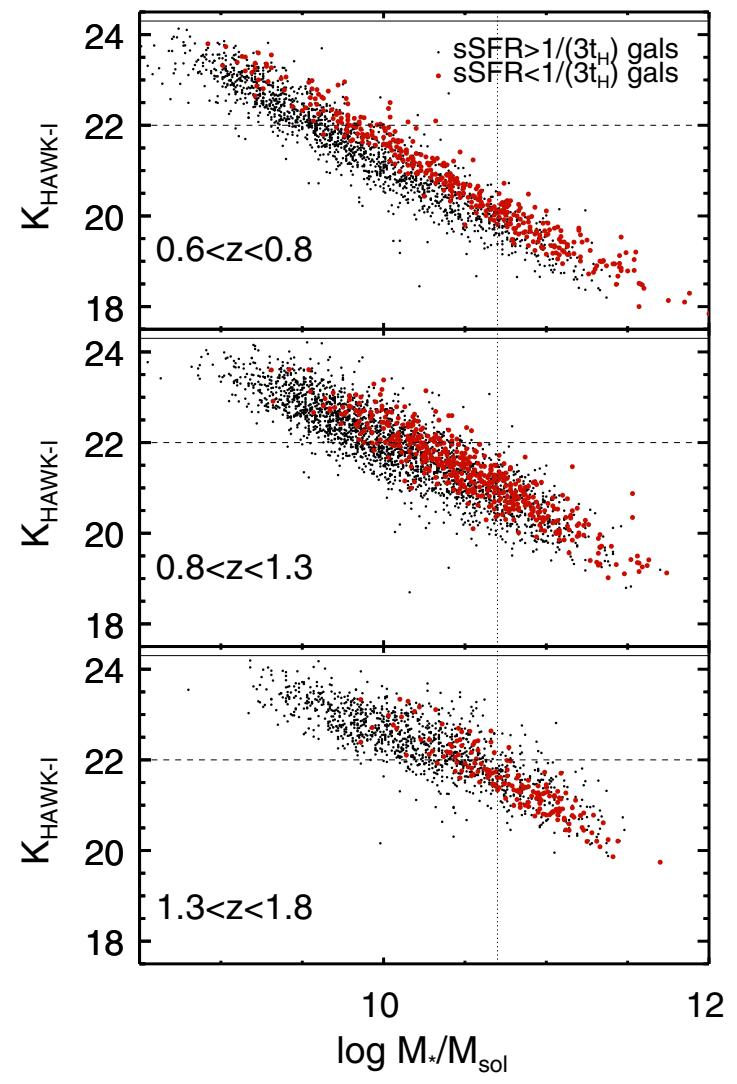

Figure 4. HAWK-I $K$-band magnitude vs. stellar mass in three redshift bins. Early-type galaxies, selected by their low sSFR (see Section 2.5.), are indicated by red symbols. The limiting factor in selecting our sample is set by the axis-ratio estimate precision (see Figure 2), which sets our magnitude limit to $K=22$ (the horizontal lines). The implication is that in our highest redshift bin we are complete down to a mass limit of $\log \left(M / M_{\odot}\right) \sim 10.7$ (the vertical lines).

(A color version of this figure is available in the online journal.)

parameters) by searching within apertures with radius $1^{\prime \prime}$. In Figure 4, we show the distribution of $K$-band magnitude as a function of stellar mass in three redshift bins. Given the magnitude limit of $K=22$ that we adopted to ensure precise axis-ratio measurements (see above), we find that our catalog is complete down to $\log \left(M / M_{\odot}\right)=10.7$ for all redshifts $z<1.8$.

We select galaxies with stellar masses $\log \left(M / M_{\odot}\right)>10.7$ and redshifts $0.6<z<1.8$, using spectroscopic redshifts when available, and, otherwise, photometric redshifts. We select as early-type galaxies those with specific star formation rates $\left(\mathrm{sSFR}=\mathrm{SFR} / M_{\odot}\right)$ smaller than $1 / 3 t_{\mathrm{H}}(z)$, where $t_{\mathrm{H}}(z)$ is the Hubble time at the photometric redshift of the galaxy. This is similar to the strategy adopted by H12, who also selected their samples by star formation activity, rather than morphological appearance.

Visual morphological classifications are not possible for the $z>1$ galaxies in our sample as they are too faint. Automated classifiers based on, for example, concentration or Sérsic index have the problem that, by definition, they will select against disk-like objects. This motivates our choice to select early-type galaxies by their star formation activity. This is justified further by the well-established agreement between morphological appearance and star formation activity at all redshifts $z<1$ (e.g., Bell et al. 2004), and the correlation between structure and star formation activity that is observed to persist out to at least $z \sim 2$ (e.g., Szomoru et al. 2011; Patel et al. 2012). 


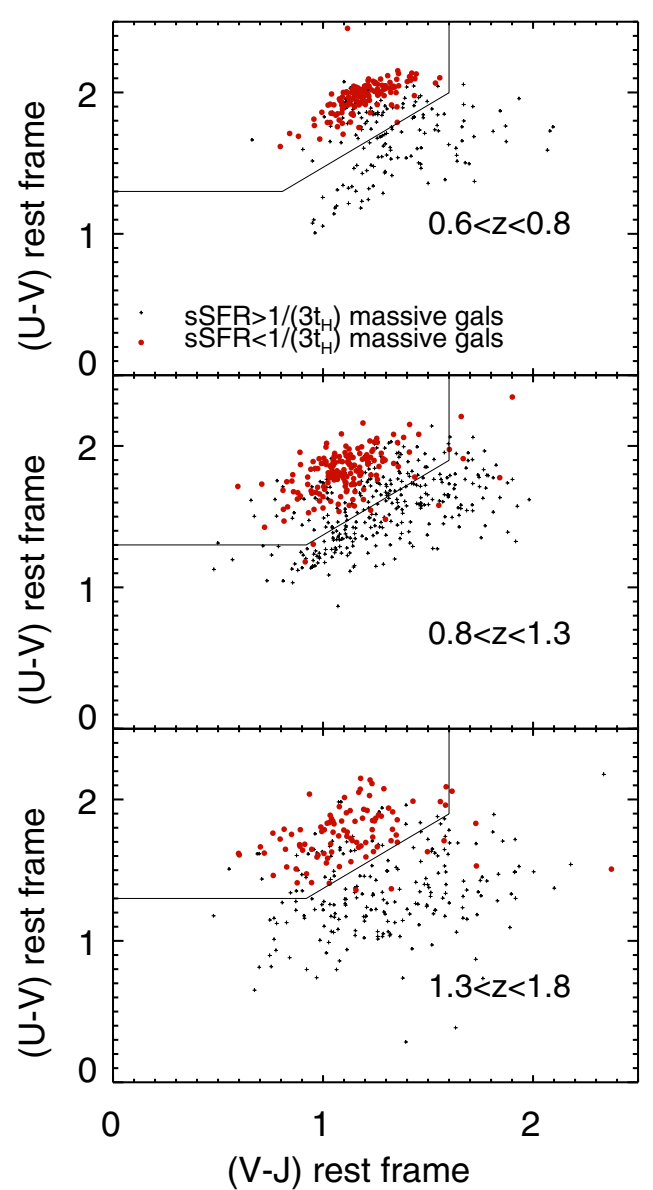

Figure 5. Rest-frame $U-V$ color vs. rest-frame $V-J$ color, a diagnostic diagram to distinguish passive and star-forming galaxies. The early-type galaxies in our sample, selected by having SED-based sSFR $(=\mathrm{SFR} / M)<1 / 3 t_{\mathrm{H}}(z)$, are indicated by red symbols. These are essentially always located in the "passive box" as defined by Williams et al. (2009) as indicated by the polygons. That our selection criteria are conservative is indicated by the presence of a substantial number of objects with $U-V$ and $V-J$ colors consistent with those of passive galaxies but with sSFR larger than our limit.

(A color version of this figure is available in the online journal.)

In Figure 5, we show that our selection technique is compatible with the rest-frame $U V J$ selection technique that is often adopted to identify passive, early-type galaxies (e.g., Wuyts et al. 2007; Williams et al. 2009). Almost all of our low-sSFR galaxies would also be identified as early-type galaxies by selecting them in this $U V J$ diagram. There are a substantial number of galaxies in the "passive" $U V J$ color-color box that are star-forming according to our direct star formation estimate fits. This number is far larger than the number of galaxies with low SFRs that are located outside the "passive" UVJ color box. While we include the latter in our subsequent analysis, we note that this does not affect our results. Samples selected by $U V J$, star formation rate, or a combination all have the same median axis ratio within 0.05 .

In Figure 6, we show the correlation between rest-frame $U-V$ color and sSFR. Even in our highest redshift bin, the populations of star-forming and passive galaxies separate cleanly, which implies little cross-contamination between the two types of galaxies.

Finally, we visually inspected all GALFIT fitting results, rejecting 10 objects with corrupted fits. The final sample consists of 394 galaxies (see Tables 1-2) in the redshift range

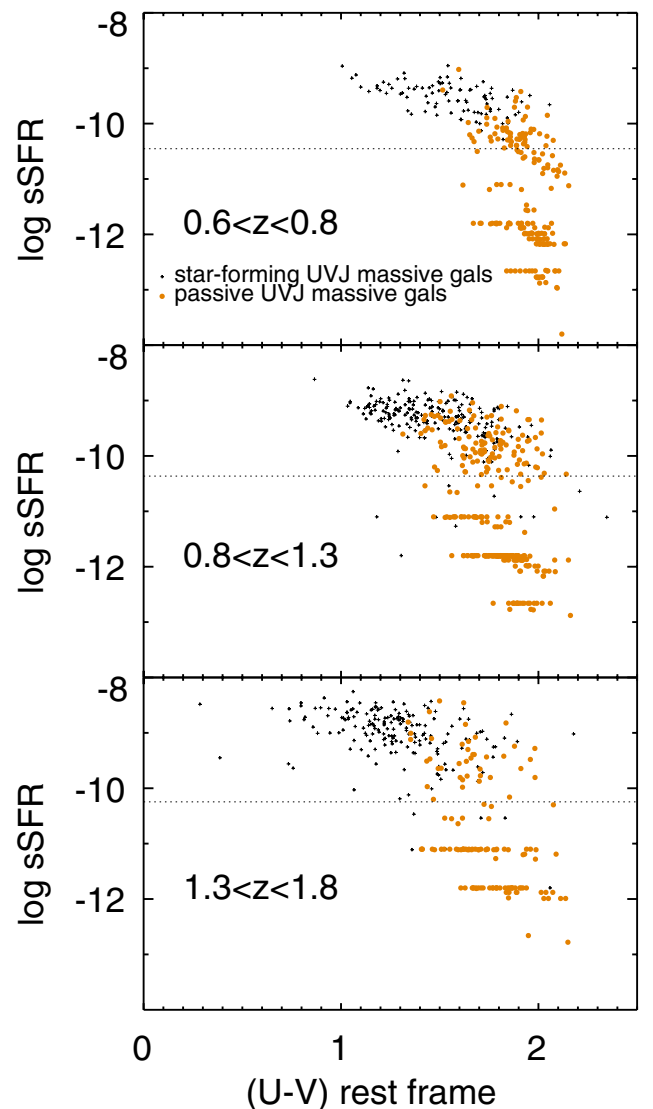

Figure 6. Correlation between rest-frame $U-V$ color and sSFR for massive galaxies in the three redshift bins. The red symbols represent galaxies in the "passive" UVJ color-color box (see Figure 5); the black symbols represent star-forming galaxies. Our selection criterion for early-type galaxies is based on an sSFR cut (sSFR $\left.<1 / 3 t_{\mathrm{H}}(z)\right)$, here illustrated by the horizontal, dotted lines which correspond to the sSFR cuts in the center of the redshift bins. For all redshift bins, the two types of galaxies are neatly separated; moving the sSFR cut by a modest amount does not strongly affect our selection of early-type galaxies. Note that aliasing occurs for low-sSFR objects as a result of template choices in the SED fitting.

(A color version of this figure is available in the online journal.)

Table 1

Massive Early-type Galaxies: $0.6<z<1.8$,

$\log \left(M / M_{\odot}\right)>10.7$, and sSFR $<1 / 3 t_{\mathrm{H}}(z)$

\begin{tabular}{lccccc}
\hline \hline ID & $z_{\text {phot }}$ & $\begin{array}{c}M \\
\left(\log M_{\odot}\right)\end{array}$ & $\begin{array}{c}K \\
(\mathrm{mag})\end{array}$ & $R_{e}$ & $q_{\text {proj }}$ \\
\hline 4032 & 1.15 & 10.72 & 20.68 & 0.25 & 0.49 \\
4213 & 1.11 & 10.73 & 20.25 & 0.35 & 0.83 \\
4619 & 1.00 & 10.87 & 21.72 & 0.09 & 0.87 \\
4844 & 1.59 & 10.94 & 20.94 & 0.35 & 0.56 \\
5375 & 1.13 & 11.33 & 19.46 & 0.44 & 0.96 \\
\hline
\end{tabular}

Notes. ID is the same as in Cardamone et al. (2010). Redshift $\left(z_{\text {phot }}\right)$ and stellar mass $(M)$ are from SED fitting. $K$-band magnitude $(K)$, effective radius $\left(R_{e}\right)$, and projected axis ratio $\left(q_{\text {proj }}\right)$ are from the GALFIT results.

(This table is available in its entirety in a machine-readable form in the online journal. A portion is shown here for guidance regarding its form and content.)

$0.6<z<1.8, \log \left(M / M_{\odot}\right)>=10.7$, and $\mathrm{sSFR}<1 / 3 t_{\mathrm{H}}(z)$. There are 134, 163, and 97 galaxies in the redshift bins, $0.6<z<0.8,0.8<z<1.3$, and $1.3<z<1.8$, respectively. 
Table 2

Statistical Properties of $q_{\text {proj }}$

\begin{tabular}{lrcccc}
\hline \hline $\log \left(M / M_{\odot}\right)>10.7$ & Number & Median & Mean & Std. dev. & Median of $z$ \\
\hline $1.53<z<2.31(\mathrm{vdW} 11)$ & 14 & 0.665 & 0.681 & 0.186 & 1.69 \\
$1.3<z<1.8$ & 97 & 0.634 & 0.612 & 0.200 & 1.38 \\
$0.8<z<1.3$ & 163 & 0.646 & 0.619 & 0.213 & 1.09 \\
$0.6<z<0.8$ & 134 & 0.734 & 0.662 & 0.222 & 0.69 \\
$0.6<z<0.8(\mathrm{H} 12)$ & 533 & 0.710 & 0.692 & 0.179 & 0.69 \\
$0.04<z<0.08(\mathrm{vdW} 09)$ & 18316 & 0.719 & 0.692 & 0.181 & 0.06 \\
\hline
\end{tabular}

\section{STRUCTURAL EVOLUTION OF MASSIVE EARLY-TYPE GALAXIES}

We now examine two aspects of the structural evolution of early-type galaxies. First, we address the question of whether the most massive galaxies are intrinsically round at redshifts $1<z<2$, as is observed at $z<1$ by vdW09 and H12. Second, we address the question whether, in general, early-type galaxies are flatter or rounder (e.g., disk-like or bulge-like) at $z>1$ than at $z<1$; H12 showed that there is little evolution up to $z<1$, but vdW11 presented tentative evidence for a higher incidence of disk-like early-type galaxies at $z \sim 2$.

\subsection{The Mass Dependence of Early-type Galaxy Shapes up to $z \sim 2$}

In Figure 7, we show the projected axis ratios of our sample of early-type galaxies as a function of their stellar mass, split into three redshift bins, each with $\sim 100$ galaxies.

Qualitatively speaking, Figure 7 shows the same trend in all redshift bins: there appears to be a mass dependence for the projected axis ratio, like that seen in vdW09. As previously demonstrated by $\mathrm{H} 12$, at $z \sim 0.7$ we find that the most massive $\left(\log \left(M / M_{\odot}\right)>11.3\right)$ early-type galaxies are round in projection and we find a lack of objects that are flat in projection among the most massive early-type galaxies (also see Huertes-Company et al. 2012). In Figure 7, a similar trend is seen for early-type galaxies at $z>1$ : at and below masses of $\log \left(M / M_{\odot}\right) \sim 11$ the galaxies in our sample show a broad range in axis ratios, whereas more massive galaxies are predominantly round. This is consistent with the conclusion reached by Targett et al. (2011), who found that very luminous radio galaxies at $z \sim 2$ have morphological properties similar to today's most massive elliptical galaxies in clusters.

In Figure 8, we compare the axis-ratio distributions of galaxies with masses below and above $\log \left(M / M_{\odot}\right) \sim 11.3$. The Mann-Whitney $U$ test shows that the trend seen in Figure 7 is marginally significant in each of the redshift bins. For the combined sample of galaxies at redshifts $0.8<z<1.8$, we find that the most massive galaxies are rounder than the less massive galaxies with high confidence $\left(P=2.51 \times 10^{-4}\right.$, i.e., a $3.66 \sigma$ result). For comparison, the equivalent value from the classical Kolmogorov-Smirnov test is also low $\left(P_{\mathrm{KS}}=1.81 \times 10^{-3}\right.$, i.e., a $3.12 \sigma$ result).

Combining the results from vdW09, H12 and from this paper, we conclude that at all redshifts $0<z<2$ the most massive early-type galaxies are predominantly round in projection. This implies that at all these cosmological epochs the formation mechanism for such massive galaxies precludes the formation of or requires the destruction of pre-existing disks. Our interpretation is that merging, accompanied with little dissipation and star formation, has been the dominant formation channel for galaxies more massive than $\log \left(M / M_{\odot}\right)=11.3$,

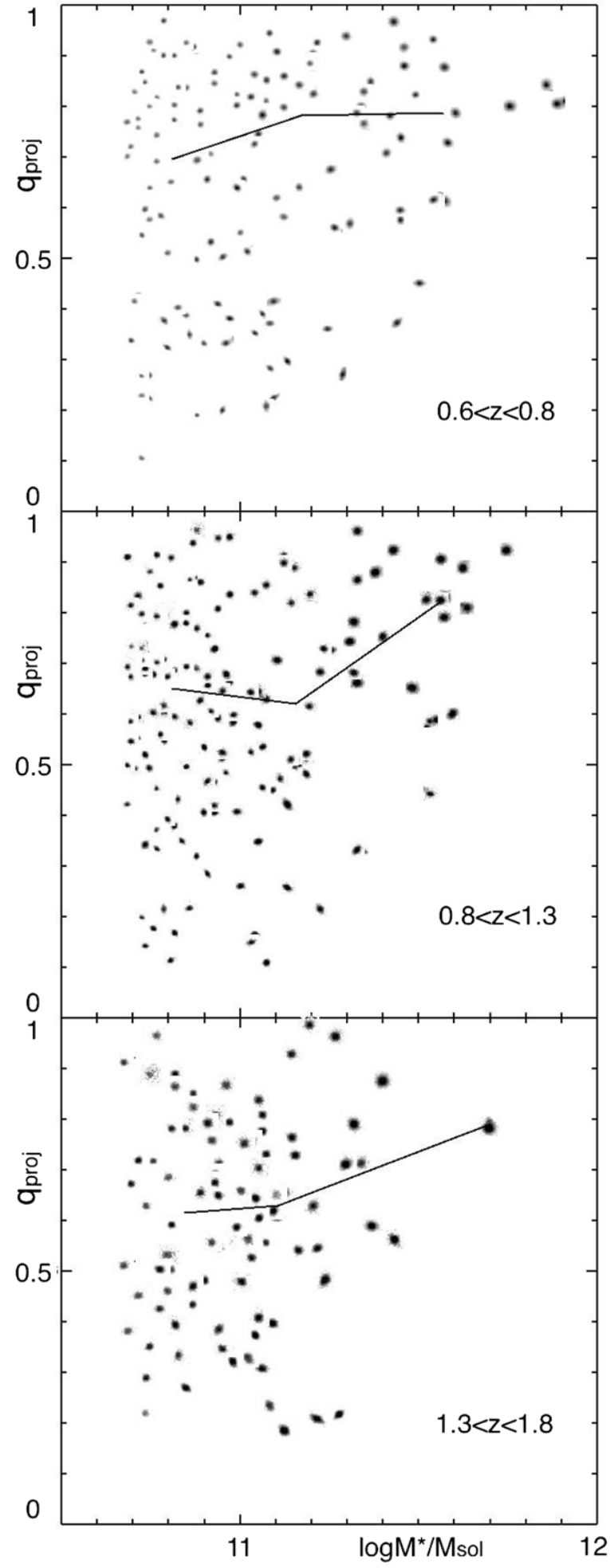

Figure 7. Projected axis ratio vs. stellar mass for our early-type galaxy sample, split into three redshift bins. The symbols are the HAWK-I $K$-band images of the galaxies. The lines represent running median values of the axis ratio. Up to our highest redshift bin, the most massive galaxies appear to be the roundest (see also Figure 8 and Section 3.1). Overall, the galaxies in the highest redshift bins are also flatter than their lower redshift counterparts (see also Figure 9 and Section 3.2).

since $z \sim 2$. This process is reproduced in early-type galaxy formation models (e.g., Naab et al. 2009; Oser et al. 2012), and the observed major merger rates up to $z \sim 2$ are consistent with theoretical expectations (e.g., Man et al. 2012). 


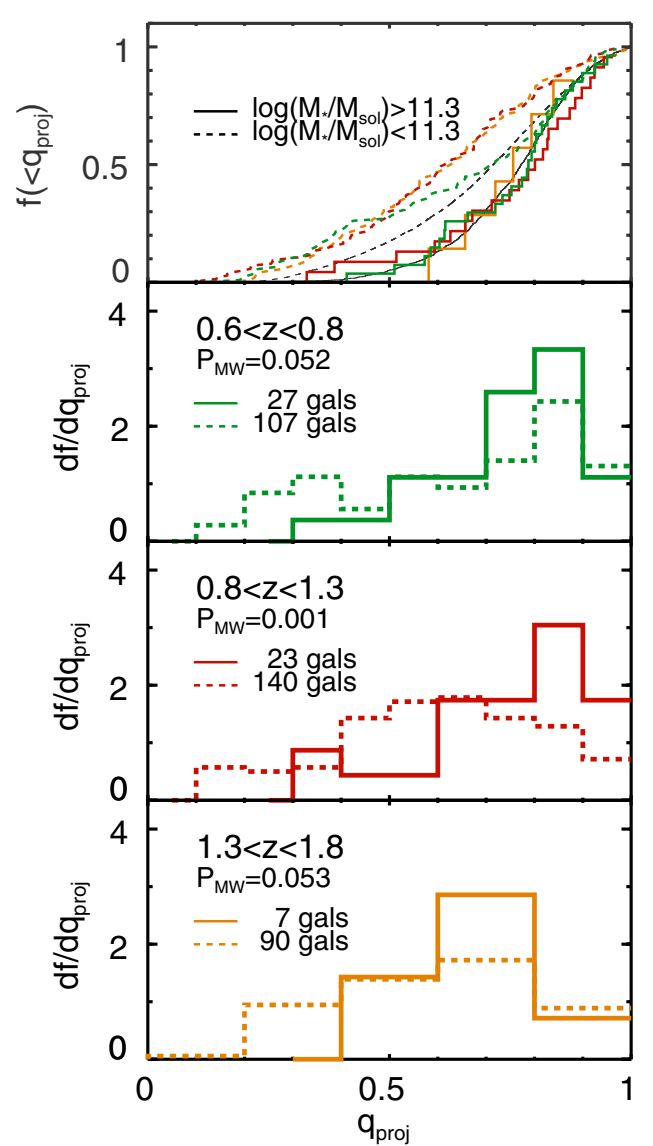

Figure 8. Axis-ratio histograms of early-type galaxies with masses $10.7<$ $\log \left(M / M_{\odot}\right)<11.3$ (dashed lines) and early-type galaxies with masses $\log \left(M / M_{\odot}\right)>11.3$ (solid lines). The bottom panels show the distributions ( $d f / d q_{\text {proj }}$ ) of different redshift bins, as indicated; the top panel combines all redshift bins and shows the cumulative histograms as a function of projected axis ratio ( $f\left(<q_{\text {proj }}\right)$, where the color coding corresponds to redshift, following the color coding in the bottom panels. The top panel also shows in black the SDSSbased sample of local early-type galaxies from $\mathrm{H} 12$ in the same mass range. At all redshifts the most massive galaxies are the roundest; the significance of this observation is confirmed by the M-W statistical test (as indicated by the listed probabilities that the samples are statistically the same).

(A color version of this figure is available in the online journal.)

\subsection{Shape Evolution at $1<z<2$}

Now we turn to the question of whether the structural population properties evolve with redshift. VdW11 found an indication of a high incidence of disk-like early-type galaxies at $z \sim 2$, but their sample is too small to confirm or rule out evolution with respect to the structure of early-type galaxies at the present day. In Figure 9, we compare the shape distributions of early-type galaxies with masses of $\log \left(M / M_{\odot}\right)>10.7$ across a broad range in redshift, $0<z<1.8$. We reproduce the lack of strong evolution in the range $0<z<0.8$, reported by $\mathrm{H} 12$.

Vulcani et al. (2011) showed that cluster early-type galaxies are rounder at $z=0.5-1$ than in the local universe. This result is not necessarily at odds with our measurements; their signal is mostly driven by galaxies below our mass limit and, moreover, structural differences between cluster and field galaxies (e.g., van der Wel et al. 2010) can be explained by environmental processes such as gas stripping which can produce flat early-type galaxies in clusters. An indication that the chosen mass range is relevant is that low-mass galaxies show stronger environmental dependencies in their properties than high-mass galaxies (see, e.g., Peng et al. 2010b). Observations over a broader range of

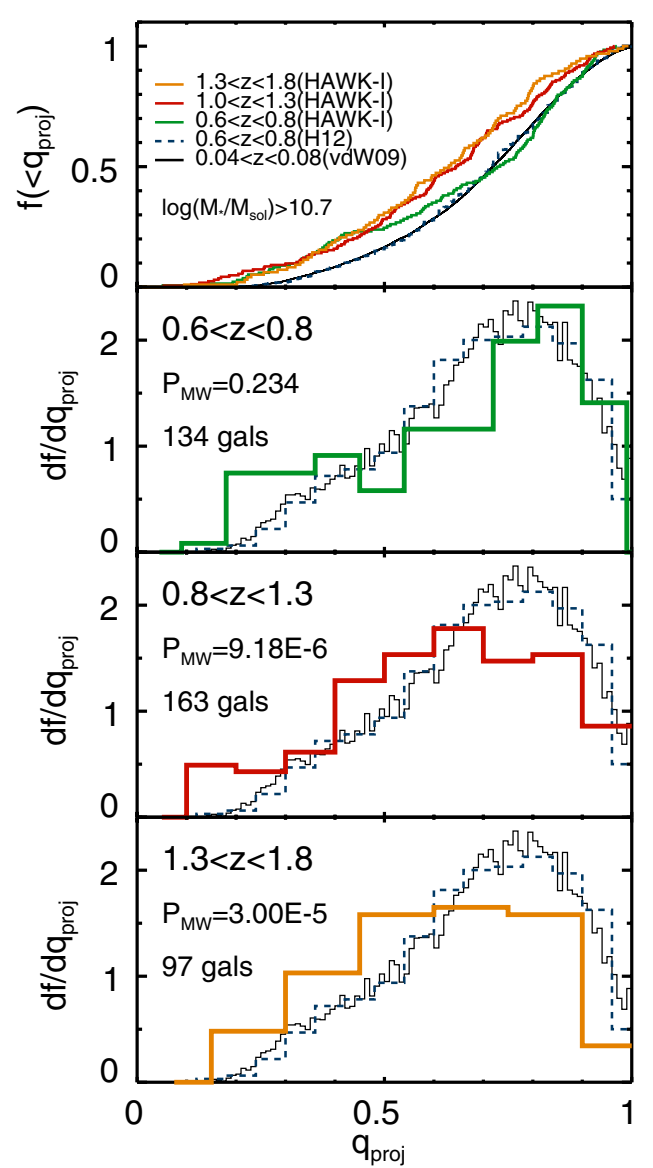

Figure 9. Axis-ratio histograms $\left(d f / d q_{\text {proj }}\right)$ of early-type galaxies with all masses $\log \left(M / M_{\odot}\right)>10.7$ (our full sample) as a function of redshift in bottom three panels, always compared with the present-day sample from H12. The top panel shows cumulative histograms for all redshift bins as a function of projected axis ratio $f\left(<q_{\text {proj }}\right)$. Up to $z=0.8$, as confirmed by the M-W statistical test (of which the probability is given that the samples are statistically the same), there is no significant redshift evolution in the projected axis-ratio distribution, consistent with the results from H12. At $z>1$, we find that early-type galaxies are flatter than their present-day counterparts.

(A color version of this figure is available in the online journal.)

environments and down to lower stellar masses than what is possible with our data set are required to address these issues.

However, in our higher redshift bins $(z>0.8)$ we see an excess of flat galaxies and a detectable evolution compared to the $z<0.8$ samples. The Kolmogorov-Smirnov (K-S) and Mann-Whitney $U(\mathrm{M}-\mathrm{W})$ tests show that this trend is highly significant (see Tables 3 and 4). Note that the axis-ratio distribution from vdW11, due to their small sample size, is consistent with both the low- and high- $z$ axis-ratio distributions we analyze here.

Figure 10 summarizes the observed evolution in the mean projected shape of early-type galaxies. Beyond $z \sim 1$, we see a gradual decrease in the median axis ratio of early-type galaxies, which is the result of an increased fraction of galaxies with axis ratios smaller than 0.6. The observed axis-ratio distributions imply that the intrinsic thickness of the typical early-type galaxy is no more than about 0.4 . In a forthcoming paper, we will quantify this intrinsic shape through detailed modeling.

The observations presented here add support to the claim by vdW11 that many high-redshift early-type galaxies display a disk-like structure and that, plausibly, these galaxies will grow into larger, intrinsically round galaxies through merging. The vdW11 sample had been too small to confirm or rule 
Table 3

Kolmogorov-Smirnov Test: Significance Level $\left(\log \left(M / M_{\odot}\right)>10.7\right)$

\begin{tabular}{|c|c|c|c|c|c|c|}
\hline $\log \left(M / M_{\odot}\right)>10.7$ & $1.53<z<2.31$ & $1.3<z<1.8$ & $0.8<z<1.3$ & $0.6<z<0.8$ & $0.6<z<0.8$ & $0.04<z<0.08$ \\
\hline $1.53<z<2.31(\mathrm{vdW} 11)$ & $\cdots$ & 0.463 & 0.549 & 0.671 & 0.668 & 0.688 \\
\hline $1.3<z<1.8$ & 0.463 & $\cdots$ & 0.875 & $1.02 \mathrm{E}-2 *$ & $2.58 \mathrm{E}-3^{*}$ & $1.54 \mathrm{E}-4 *$ \\
\hline $0.8<z<1.3$ & 0.549 & 0.875 & $\ldots$ & $1.26 \mathrm{E}-2 *$ & $8.93 \mathrm{E}-4^{*}$ & $1.21 \mathrm{E}-4^{*}$ \\
\hline $0.6<z<0.8$ & 0.671 & $1.02 \mathrm{E}-2 *$ & $1.26 \mathrm{E}-2^{*}$ & $\ldots$ & 0.060 & 0.035 \\
\hline $0.6<z<0.8(\mathrm{H} 12)$ & 0.668 & $2.58 \mathrm{E}-3^{*}$ & $8.93 \mathrm{E}-4^{*}$ & 0.060 & $\cdots$ & 0.584 \\
\hline $0.04<z<0.08(\mathrm{vdW} 09)$ & 0.688 & $1.54 \mathrm{E}-3 *$ & $1.21 \mathrm{E}-4 *$ & 0.035 & 0.584 & $\cdots$ \\
\hline
\end{tabular}

Note. The asterisk $\left(^{*}\right)$ indicates that the significant probability is smaller than $5 \%$. This implies that the distributions are distinguishable.

Table 4

Mann-Whitney Test: Significance Level $\left(\log \left(M / M_{\odot}\right)>10.7\right)$

\begin{tabular}{|c|c|c|c|c|c|c|}
\hline $\log \left(M / M_{\odot}\right)>10.7$ & $1.53<z<2.31$ & $1.3<z<1.8$ & $0.8<z<1.3$ & $0.6<z<0.8$ & $0.6<z<0.8$ & $0.04<z<0.08$ \\
\hline $1.53<z<2.31(\mathrm{vdW} 11)$ & $\cdots$ & 0.139 & 0.167 & 0.456 & 0.372 & 0.373 \\
\hline $1.3<z<1.8$ & 0.139 & $\ldots$ & 0.315 & $1.12 \mathrm{E}-2 *$ & $1.20 \mathrm{E}-4 *$ & $3.00 \mathrm{E}-5^{*}$ \\
\hline $0.8<z<1.3$ & 0.167 & 0.315 & $\ldots$ & $2.06 \mathrm{E}-2 *$ & $8.71 \mathrm{E}-5^{*}$ & $9.18 \mathrm{E}-6^{*}$ \\
\hline $0.6<z<0.8$ & 0.456 & $1.12 \mathrm{E}-2 *$ & $2.06 \mathrm{E}-2^{*}$ & $\ldots$ & 0.249 & 0.234 \\
\hline $0.6<z<0.8(\mathrm{H} 12)$ & 0.372 & $1.20 \mathrm{E}-4^{*}$ & $8.71 \mathrm{E}-5^{*}$ & 0.249 & $\ldots$ & 0.445 \\
\hline $0.04<z<0.08$ (vdW09) & 0.381 & $3.00 \mathrm{E}-5^{*}$ & $9.18 \mathrm{E}-6^{*}$ & 0.234 & 0.445 & $\ldots$ \\
\hline
\end{tabular}

Note. The asterisk $(*)$ indicates that the significant probability is smaller than $5 \%$. This implies that the distributions are distinguishable.

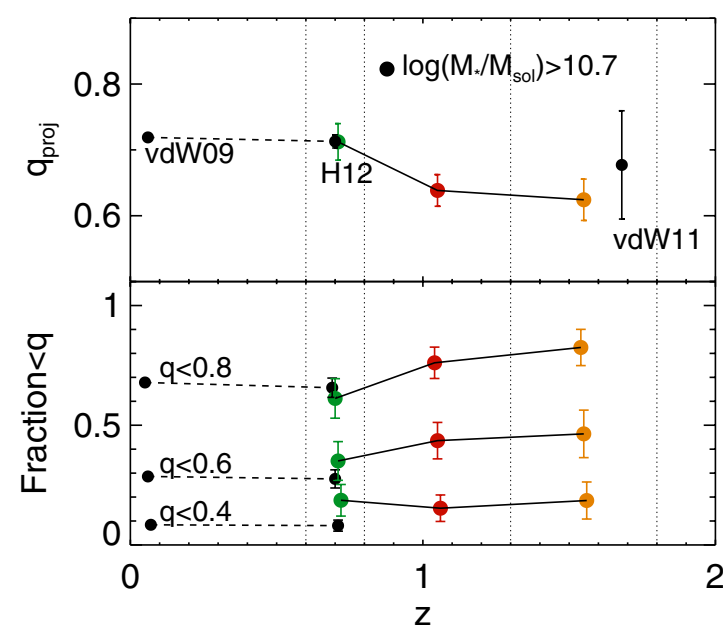

Figure 10. Top panel: median axis ratio of early-type galaxies more massive than $\log \left(M / M_{\odot}\right)>10.7$ as a function of redshift. The sample presented in this paper is represented by the colored data points; the black data points are taken from the literature as indicated. The error bars are estimated by bootstrapping. Bottom panel: fraction of galaxies with axis ratios smaller than indicated, again as a function of redshift. Beyond $z \sim 1$, early-type galaxies typically have smaller axis ratios than present-day counterparts in the same mass range, indicative of a more disk-like structure. All error bars are inferred by the $95 \%$ confidence level compared to the same fraction.

(A color version of this figure is available in the online journal.)

evolution at a fixed stellar mass, which is important in order to differentiate evolutionary paths that galaxies of a given mass take at different cosmic times. Even at the present day, the typical early-type galaxy with a mass below $\log \left(M / M_{\odot}\right)=11$ is rather flattened (vdW09), which also corresponds to a disklike kinematic structure (rotation) as shown, for example, by Emsellem et al. (2011). Here we show that such galaxies had even thinner intrinsic shapes at $z>1$. Good correspondence between rotation of the stellar body and flattening has been confirmed up to $z \sim 1$ by van der Wel \& van der Marel (2008). Although such confirmation is, so far, lacking at higher redshifts, it is reasonable to assume that flattening implies rotation at all redshifts.
We note that beyond the simple observation that early-type galaxies, defined here as galaxies with little star formation activity, are on average flatter at $z>1$ than at lower redshifts, we cannot distinguish between the different varieties of such galaxies — such as quiescent spirals and barred/ringed SOs_-and their evolution.

Our results do not argue against the occurrence of a morphological transition along with the truncation of star formation, as argued by Bell et al. (2012), for example, on the basis of the high Sérsic indices of quiescent galaxies: high-redshift earlytype galaxies such as those studied here, despite the generally disk-like character inferred from their axis-ratio distribution, are not pure disks and have higher Sérsic indices than star-forming galaxies (e.g., Wuyts et al. 2011b). Based on bulge-disk decompositions, Bruce et al. (2012) arrive at the conclusion that the early-type galaxy population at $z>1$ is a mix of bulge- and disk-dominated galaxies, indicating that the transition from actively star-forming to quiescent need not always coincide with the formation of a dominant bulge. Taken together, these observations are consistent with a picture in which gas had time to settle in a disk before star formation was truncated, and that this disk wholly or partially survived the process that truncated star formation.

\section{CONCLUSIONS}

We measured the projected axis ratios from VLT/HAWK-I $K$-band imaging of a sample of early-type galaxies in the redshift range $0.6<z<1.8$ selected by their low sSFRs. We found that at all redshifts $z<2$ the most massive galaxies $\log \left(M / M_{\odot}\right)>11.3$ are predominantly round. The lack of very massive, highly flattened galaxies suggests a universal ceiling mass for the formation of disks, independent of cosmic epoch. In order for galaxies to grow beyond this ceiling mass, a separate evolutionary channel, presumably merging, has to be invoked.

In the full sample $\left(\log \left(M / M_{\odot}\right)>10.7\right)$, we find, at all redshifts, a large range in projected axis ratios, reflecting a more disk-like structure. In addition, we find quantitative evidence that early-type galaxies at $z>1$ are more disk-like than 
their equally massive, present-day counterparts. Therefore, for most early-type galaxies, gas had time to settle into a disk before star formation ceased to produce a more passive galaxy. Plausibly, such galaxies grow in mass over time, through mostly dissipationless merging and accretion of satellites, losing some of their angular momentum, and growing in size.

Y.-Y. Chang was funded by the IMPRS for Astronomy $\&$ Cosmic Physics at the University of Heidelberg and the Marie Curie Initial Training Network ELIXIR of the European Commission under contract PITN-GA-2008-214227.

\section{REFERENCES}

Barden, M., Häußler, B., Peng, C. Y., McIntosh, D. H., \& Guo, Y. 2012, MNRAS, 422, 449

Bell, E. F., van der Wel, A., Papovich, C., et al. 2012, ApJ, 753, 167

Bell, E. F., Wolf, C., Meisenheimer, K., et al. 2004, ApJ, 608, 752

Benson, A. J., Džanović, D., Frenk, C. S., \& Sharples, R. 2007, MNRAS, 379,841

Bernardi, M., Roche, N., Shankar, F., \& Sheth, R. K. 2011, MNRAS, 412, 684

Bertin, E., \& Arnouts, S. 1996, A\&AS, 117, 393

Bezanson, R., van Dokkum, P. G., Tal, T., et al. 2009, ApJ, 697, 1290

Brammer, G. B., van Dokkum, P. G., \& Coppi, P. 2008, ApJ, 686, 1503

Bruce, V. A., Dunlop, J. S., Cirasuolo, M., et al. 2012, MNRAS, in press (arXiv:1206.4322)

Bruzual, G., \& Charlot, S. 2003, MNRAS, 344, 1000

Buitrago, F., Trujillo, I., Conselice, C. J., \& Haeussler, B. 2012, MNRAS, in press (arXiv:1111.6993)

Cardamone, C. N., van Dokkum, P. G., Urry, C. M., et al. 2010, ApJS, 189, 270

Chabrier, G. 2003, PASP, 115, 763

Cheng, J. Y., Faber, S. M., Simard, L., et al. 2011, MNRAS, 412, 727

Chevance, M., Weijmans, A.-M., Damjanov, I., et al. 2012, ApJ, 754, L24

Dressler, A. 1980, ApJ, 236, 351

Emsellem, E., Cappellari, M., Krajnović, D., et al. 2011, MNRAS, 414, 888

Faber, S. M., Willmer, C. N. A., Wolf, C., et al. 2007, ApJ, 665, 265

Häussler, B., McIntosh, D. H., Barden, M., et al. 2007, ApJS, 172, 615

Huertes-Company, M., Mei, S., Shankar, F., et al. 2012, MNRAS, in press (arXiv:1207.5793)

Holden, B. P., van der Wel, A., Rix, H.-W., \& Franx, M. 2012, ApJ, 749, 96

Holden, B. P., Franx, M., Illingworth, G. D., et al. 2009, ApJ, 693, 617

Hopkins, P. F., Bundy, K., Hernquist, L., Wuyts, S., \& Cox, T. J. 2010, MNRAS, 401, 1099

Jorgensen, I., \& Franx, M. 1994, ApJ, 433, 553

Joseph, R. D., \& Wright, G. S. 1985, MNRAS, 214, 87
Kauffmann, G., White, S. D. M., \& Guiderdoni, B. 1993, MNRAS, 264, 201

Khochfar, S., \& Silk, J. 2006, MNRAS, 370, 902

Kormendy, J., \& Djorgovski, S. 1989, ARA\&A, 27, 235

Krajnović, D., Bacon, R., Cappellari, M., et al. 2008, MNRAS, 390, 93

Kriek, M., van Dokkum, P. G., Labbé, I., et al. 2009, ApJ, 700, 221

Magnelli, B., Elbaz, D., Chary, R. R., et al. 2009, A\&A, 496, 57

Man, A. W. S., Toft, S., Zirm, A. W., Wuyts, S., \& van der Wel, A. 2012, ApJ, 744,85

Marinoni, C., Monaco, P., Giuricin, G., \& Costantini, B. 1999, ApJ, 521, 50

McLure, R. J., Pearce, H. J., Dunlop, J. S., et al. 2012, MNRAS, in press (arXiv:1205.4058)

Naab, T., Johansson, P. H., \& Ostriker, J. P. 2009, ApJ, 699, L178

Oser, L., Naab, T., Ostriker, J. P., \& Johansson, P. H. 2012, ApJ, 744, 63

Patel, S. G., van Dokkum, P. G., Franx, M., et al. 2012, ApJ, submitted (arXiv:1208.0341)

Peng, C. Y., Ho, L. C., Impey, C. D., \& Rix, H.-W. 2002, AJ, 124, 266

Peng, C. Y., Ho, L. C., Impey, C. D., \& Rix, H.-W. 2010a, AJ, 139, 2097

Peng, Y.-j., Lilly, S. J., Kovač, K., et al. 2010b, ApJ, 721, 193

Rix, H.-W., Barden, M., Beckwith, S. V. W., et al. 2004, ApJS, 152, 163

Robaina, A. R., Bell, E. F., van der Wel, A., et al. 2010, ApJ, 719, 844

Schweizer, F., Seitzer, P., Faber, S. M., et al. 1990, ApJ, 364, L33

Steinmetz, M., \& Navarro, J. F. 2002, New Astron., 7, 155

Szomoru, D., Franx, M., Bouwens, R. J., et al. 2011, ApJ, 735, L22

Targett, T. A., Dunlop, J. S., McLure, R. J., et al. 2011, MNRAS, 412, 295

Taylor, E. N., Franx, M., van Dokkum, P. G., et al. 2009, ApJS, 183, 295

Toomre, A. 1977, ARA\&A, 15, 437

van der Wel, A., Bell, E. F., Häussler, B., et al. 2012, ApJS, 203, 24

van der Wel, A., Bell, E. F., Holden, B. P., Skibba, R. A., \& Rix, H.-W. 2010, ApJ, 714,1779

van der Wel, A., Bell, E. F., van den Bosch, F. C., Gallazzi, A., \& Rix, H.-W. 2009a, ApJ, 698, 1232

van der Wel, A., Holden, B. P., Zirm, A. W., et al. 2008, ApJ, 688, 48

van der Wel, A., Rix, H.-W., Holden, B. P., Bell, E. F., \& Robaina, A. R. 2009b, ApJ, 706, L120

van der Wel, A., Rix, H.-W., Wuyts, S., et al. 2011, ApJ, 730, 38

van der Wel, A., \& van der Marel, R. P. 2008, ApJ, 684, 260

van Dokkum, P. G., Franx, M., Kriek, M., et al. 2008, ApJ, 677, L5

Vincent, R. A., \& Ryden, B. S. 2005, ApJ, 623, 137

Vulcani, B., Poggianti, B. M., Dressler, A., et al. 2011, MNRAS, 413, 921

Whitaker, K. E., Kriek, M., van Dokkum, P. G., et al. 2012, ApJ, 745, 179

White, S. D. M., \& Rees, M. J. 1978, MNRAS, 183, 341

Williams, R. J., Quadri, R. F., Franx, M., van Dokkum, P., \& Labbé, I. 2009, ApJ, 691, 1879

Windhorst, R. A., Cohen, S. H., Hathi, N. P., et al. 2011, ApJS, 193, 27

Wuyts, S., Labbé, I., Franx, M., et al. 2007, ApJ, 655, 51

Wuyts, S., Förster Schreiber, N. M., Lutz, D., et al. 2011a, ApJ, 738, 106

Wuyts, S., Förster Schreiber, N. M., van der Wel, A., et al. 2011b, ApJ, 742,96 